\title{
$\phi$ meson in nuclear matter and nuclei
}

\author{
J. J. Cobos-Martínez ${ }^{\star a b}$, K. Tsushima ${ }^{b}$, G. Krein ${ }^{c}$, and A. W. Thomas ${ }^{d}$ \\ ${ }^{a}$ CONACyT-Departamento de Física, Centro de Investigación y de Estudios Avanzados del \\ Instituto Politécnico Nacional, Apartado Postal 14-740, 07000, Ciudad de México, México. \\ ${ }^{b}$ Laboratório de Física Teórica e Computacional-LFTC, Universidade Cruzeiro do Sul, \\ 01506-000, São Paulo, SP, Brazil. \\ ${ }^{c}$ Instituto de Física Teórica, Universidade Estadual Paulista, Rua Dr. Bento Teobaldo Ferraz, \\ 271-Bloco II, 01140-070, São Paulo, SP, Brazil. \\ ${ }^{d}$ ARC Centre of Excellence for Particle Physics at the Terascale and CSSM, Department of \\ Physics, University of Adelaide, Adelaide SA 5005, Australia.
}

E-mail: jcobosefis.cinvestav.mx,kazuo.tsushima@gmail.com, gkreineift.unesp.br, anthony.thomaseadelaide.edu.au

\begin{abstract}
The mass and decay width of the $\phi$ meson in cold nuclear matter are computed in an effective Lagrangian approach, with the medium dependence of these properties obtained by evaluating kaon-antikaon loop contributions to the $\phi$ self-energy. At normal nuclear matter density, we find a downward shift of the $\phi$ mass by a few percent, while the decay width is enhanced by an order of magnitude. We also present $\phi$-nucleus bound state energies and absorption widths for some selected nuclei, using complex optical potentials obtained in the local density approximation. Our results suggest that the $\phi$ should form bound states with all the nuclei considered. However, the identification of the signal for these predicted bound states will need careful investigation because of their sizable absorption widths.
\end{abstract}

XVII International Conference on Hadron Spectroscopy and Structure

25-29 September, 2017

University of Salamanca, Salamanca, Spain

${ }^{*}$ Speaker. 


\section{Introduction}

The properties of the $\phi$ meson at finite baryon density, such as its mass and decay width, have attracted considerable experimental and theoretical interest over the last few decades [1]. This has been in part due to its potential to carry information on the partial restoration of chiral symmetry, the possible role of QCD of van der Waals forces in the binding of quarkonia to nuclei [2], and the strangeness content of the nucleon [3]. However, a experimental unified consensus has not yet been reached among the different experiments concerning the $\phi$. A large in-medium broadening of its decay width has been reported by most of the experiments performed, while only a few of them find evidence for a substantial mass shift [4]. The study of the $\phi$-nucleus bound states $[5,6]$ is complementary to the invariant mass measurements, where only a small fraction of the produced $\phi$ decay inside the nucleus, and may be expected to provide extra information on the $\phi$ properties at finite baryon density, since a downward mass shift of the $\phi$ in a nucleus is directly connected with the existence of an attractive potential between the $\phi$ and the nucleus where it has been produced. This new experimental approach will produce a slowly moving $\phi$ such that the maximum nuclear matter effect can be probed. On the theoretical side, various authors predict a small downward shift of the in-medium $\phi$ mass and a large broadening of its decay width [7] at normal nuclear matter density. An important question is whether this attraction, if it exists, is sufficient to bind the $\phi$ to a nucleus. Using simple quantum mechanical arguments, the prospects of capturing a $\phi$ seem quite favorable, provided that the $\phi$ can be produced almost at rest in the nucleus. However, a full calculation using realistic density profiles of nuclei is required for a more reliable estimate. The work presented at this conference has been published in Refs. [8, 9]. In Ref. [8] we computed the $\phi$ mass shift and decay width in nuclear matter by evaluating the $K \bar{K}$ loop contribution to the $\phi$ self-energy, with the in-medium $K$ and $\bar{K}$ masses explicitly calculated using the quark-meson coupling (QMC) model [10]. This initial study is been extended in Ref. [9] to some selected nuclei by computing the $\phi$-nucleus bound state energies and absorption with a complex optical potential calculated in the local density approximation.

\section{2. $\phi$ mass and decay width in nuclear matter}

One expects that a significant fraction of the density dependence of the $\phi$ self-energy in nuclear matter arises from the in-medium modification of the $K \bar{K}$ intermediate state in the $\phi$ self-energy, since this is the dominant decay channel in vacuum. We briefly review the computation [8] of the $\phi$ self-energy in vacuum and in nuclear matter using an effective Lagrangian approach [11]. The interaction Lagrangian contains only the $\phi K \bar{K}$ vertex, and is given by

$$
\mathscr{L}_{\phi K \bar{K}}=\mathrm{i}_{\phi} \phi^{\mu}\left[\bar{K}\left(\partial_{\mu} K\right)-\left(\partial_{\mu} \bar{K}\right) K\right],
$$

where $K$ and $\bar{K}$ are isospin doublets. The contribution from the $\phi K \bar{K}$ coupling to the scalar part of the $\phi$ self-energy, $\Pi_{\phi}(p)$, is given by

$$
\mathrm{i}_{\phi}(p)=-\frac{8}{3} g_{\phi}^{2} \int \frac{\mathrm{d}^{4} q}{(2 \pi)^{4}} \vec{q}^{2} D_{K}(q) D_{K}(q-p),
$$

where $D_{K}(q)=1 /\left(q^{2}-m_{K}^{2}+\mathrm{i} \varepsilon\right)$ is the kaon propagator; $p=\left(p^{0}=m_{\phi}, \overrightarrow{0}\right)$ for $\phi$ at rest, with $m_{\phi}$ the $\phi$ mass; $m_{K}\left(=m_{\bar{K}}\right)$ is the kaon mass, and $g_{\phi}=4.539$ [8] is the coupling constant. The integral 
in Eq. (2.2) is divergent but it will be regulated using a phenomenological form factor, with cutoff parameter $\Lambda_{K}$ [8]. The sensitivity of the results to the value of $\Lambda_{K}$ is analyzed below. The mass and decay width of the $\phi$ in vacuum $\left(m_{\phi}\right.$ and $\left.\Gamma_{\phi}\right)$, as well as in nuclear matter $\left(m_{\phi}^{*}\right.$ and $\left.\Gamma_{\phi}^{*}\right)$, are determined [8] from

$$
\begin{aligned}
m_{\phi}^{2} & =\left(m_{\phi}^{0}\right)^{2}+\mathfrak{R} \Pi_{\phi}\left(m_{\phi}^{2}\right), \\
\Gamma_{\phi} & =-\frac{1}{m_{\phi}} \mathfrak{I} \Pi_{\phi}\left(m_{\phi}^{2}\right) .
\end{aligned}
$$

The density dependence of the $\phi$ mass and decay width is driven by the intermediate $K \bar{K}$ state interactions with the nuclear medium. This effect enters through the Lorentz scalar mass $m_{K}^{*}$ in the kaon propagators in Eq. (2.2). The in-medium properties of $K$ and $\bar{K}$ are calculated in the QMC model [10] (see Ref. [12] for a review) considering infinitely large, uniformly symmetric, spin-isospin-saturated nuclear matter in its rest frame, where all the scalar and vector mean field potentials, which are responsible for the nuclear many-body interactions, become constant in the Hartree approximation. In Figure 1 (left panel) we present the resulting in-medium kaon Lorentz scalar mass (=antikaon Lorentz scalar mass) as a function of the baryon density. At normal nuclear matter density $\rho_{0}=0.15 \mathrm{fm}^{-3}$ the effective kaon mass has decreased by about $13 \%$. We recall that the isoscalar-vector $\omega$ mean field potentials arise both for the kaon and antikaon, but they can be eliminated by a variable shift in the loop calculation of the $\phi$ self-energy, and therefore are not show them here. In Figure 1, we present the $\phi$ mass shift (centre panel) and decay width (right panel) as
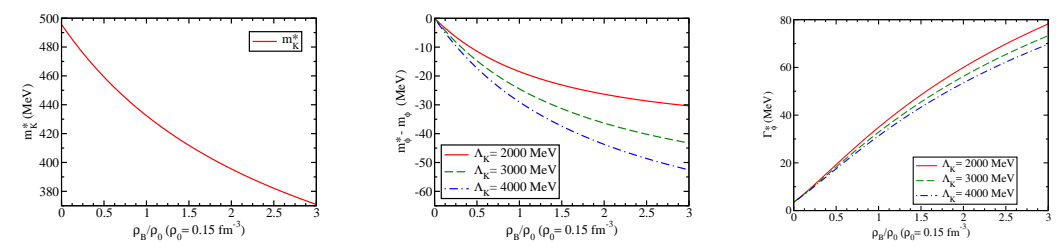

Figure 1: Left panel: In-medium Lorentz scalar kaon (=antikaon) mass $m_{K}^{*}$; centre and right panels: $\phi$ mass shift and decay width, respectively, for three values of $\Lambda_{K}$.

a function of the nuclear matter density, $\rho_{B}$, for three values of $\Lambda_{K}$. The effect of the in-medium kaon and antikaon mass change yields a negative mass shift for the $\phi$. This is because the reduction in the kaon and antikaon masses enhances the $K \bar{K}$ loop contribution in nuclear matter relative to that in vacuum. For the largest value of $\rho_{B}$, the downward mass shift turns out to be a few percent at most for all values of $\Lambda_{K}$. On the other hand, $\Gamma_{\phi}^{*}$ is very sensitive to the change in the kaon and antikaon masses, increasing rapidly with increasing nuclear matter density, up to a factor of $\approx 20$ for the largest value of $\rho_{B}$. These results open the experimental possibility for studying the binding and absorption of $\phi$ in nuclei. Although the mass shift found in this study may be large enough to bind the $\phi$ to a nucleus, the broadening of its decay width will make it difficult to observe a signal for the $\phi$-nucleus bound state formation experimentally. We explore this further in the second part of this talk. 


\section{3. $\phi$-nucleus bound states}

We now explore the situation where the $\phi$ meson is placed inside a nucleus [9]. The nuclear density distributions for all nuclei but ${ }^{4} \mathrm{He}$ are obtained in the QMC model [13]. For ${ }^{4} \mathrm{He}$, we use Ref. [14]. Then, using a local density approximation the $\phi$-nucleus potentials for a nucleus $A$ is given by

$$
V_{\phi A}(r)=U_{\phi}(r)-\frac{\mathrm{i}}{2} W_{\phi}(r),
$$

where $r$ is the distance from the center of the nucleus and $U_{\phi}(r)=m_{\phi}^{*}\left(\rho_{B}(r)\right)-m_{\phi}$ and $W_{\phi}(r)=$ $\Gamma_{\phi}\left(\rho_{B}(r)\right)$ are, respectively, the $\phi$ mass shift and decay width inside nucleus $A$, with $\rho_{B}(r)$ the baryon density distribution of the given nucleus. In Figure 2 we show the $\phi$ potentials calculated [9]
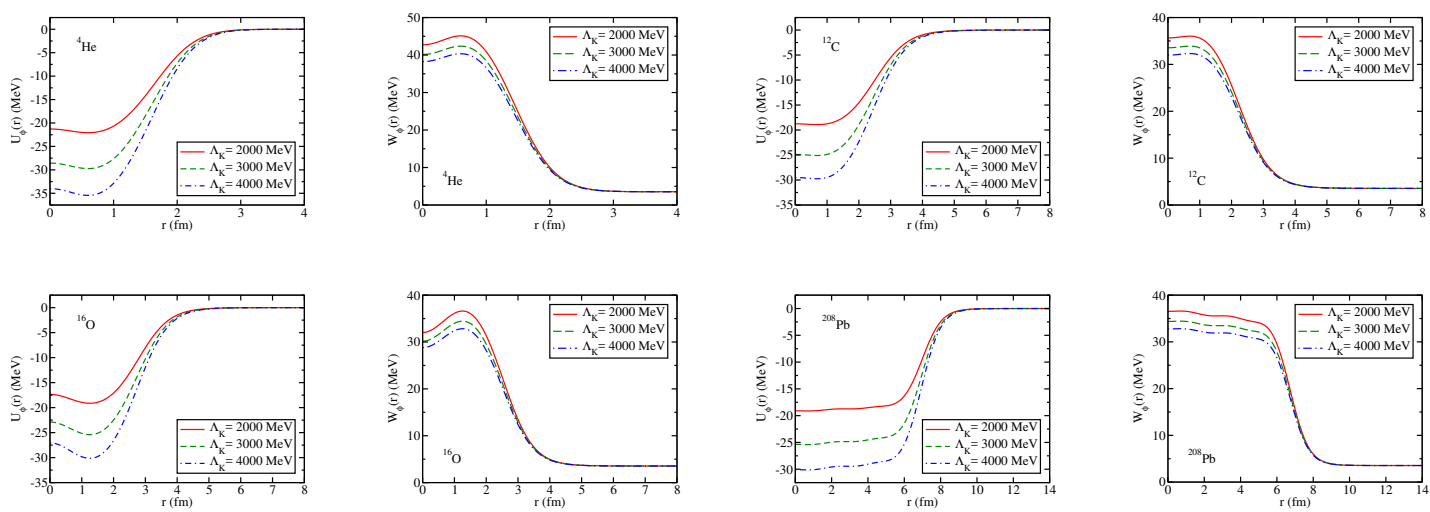

Figure 2: Real $\left[U_{\phi}(r)\right]$ and imaginary $[W \phi(r)]$ parts of the $\phi$-nucleus potentials for the nuclei studied here, for three values of $\Lambda_{K}$.

for the selected nuclei. One can see that the depth of the real part of the potential is sensitive to $\Lambda_{K}$. On the other hand, the imaginary part does not vary much with $\Lambda_{K}$. Using the $\phi$ potentials, we next calculate the $\phi$ single-particle energies and absorption widths. We consider the situation where the $\phi$ is produced nearly at rest [9]. Then, under this condition, solving the Proca equation becomes equivalent to solving the Klein-Gordon equation

$$
\left(-\nabla^{2}+\mu^{2}+2 \mu V(r)\right) \phi(\vec{r})=\mathscr{E}^{2} \phi(\vec{r}),
$$

where $\mu$ is the reduced mass of the $\phi$-nucleus system in vacuum, and $V(r)$ is given by Eq. (3.1). The calculated bound state energies $(E)$ and absorption widths $(\Gamma)$ [9], which are related to the complex energy eigenvalue $\mathscr{E}$ by $E=\mathfrak{R} \mathscr{E}-\mu$ and $\Gamma=-2 \mathfrak{I} \mathscr{E}$, are listed in Table 1 with and without the imaginary part of the potential. When the imaginary part of the $\phi$-nucleus potential is set to zero we see that the $\phi$ is expected to form bound states with all the nuclei selected. The bound state energy is obviously dependent on $\Lambda_{K}$, increasing as $\Lambda_{K}$ increases. Adding the absorptive part of the potential changes the situation considerably. We note that for the largest value of $\Lambda_{K}$, which yields the deepest potentials, the $\phi$ is expected to form bound states with all the selected nuclei, including ${ }^{4} \mathrm{He}$. However, in this case, whether or not the bound states can be observed experimentally is sensitive to the value of $\Lambda_{K}$. One also observes that the width of the bound state is insensitive to the values of $\Lambda_{K}$ for all nuclei. Furthermore, since the so-called dispersive effect 
of the absorptive potential is repulsive, the bound states disappear completely in some cases, even though they were found when the absorptive part was set to zero. This feature is obvious for the ${ }^{4} \mathrm{He}$ nucleus, making it especially relevant to the future experiments, planned at J-PARC and JLab using light and medium-heavy nuclei $[5,6]$.

\begin{tabular}{ll|rr|rr|rr}
\hline \hline & & \multicolumn{2}{|c|}{$\Lambda_{K}=2000$} & \multicolumn{2}{|c|}{$\Lambda_{K}=3000$} & \multicolumn{2}{|c}{$\Lambda_{K}=4000$} \\
\hline & & $E$ & $\Gamma / 2$ & $E$ & $\Gamma / 2$ & $E$ & $\Gamma / 2$ \\
\hline${ }_{\phi}^{4} \mathrm{He}$ & $1 \mathrm{~s}$ & $\mathrm{n}(-0.8)$ & $\mathrm{n}$ & $\mathrm{n}(-1.4)$ & $\mathrm{n}$ & $-1.0(-3.2)$ & 8.3 \\
\hline${ }_{\phi}^{12} \mathrm{C}$ & $1 \mathrm{~s}$ & $-2.1(-4.2)$ & 10.6 & $-6.4(-7.7)$ & 11.1 & $-9.8(-10.7)$ & 11.2 \\
\hline${ }_{\phi}^{16} \mathrm{O}$ & $1 \mathrm{~s}$ & $-4.0(-5.9)$ & 12.3 & $-8.9(-10.0)$ & 12.5 & $-12.6(-13.4)$ & 12.4 \\
& $1 \mathrm{p}$ & $\mathrm{n}(\mathrm{n})$ & $\mathrm{n}$ & $\mathrm{n}(\mathrm{n})$ & $\mathrm{n}$ & $\mathrm{n}(-1.5)$ & $\mathrm{n}$ \\
\hline${ }_{\phi}^{208} \mathrm{~Pb}$ & $1 \mathrm{~s}$ & $-15.0(-15.5)$ & 17.4 & $-21.1(-21.4)$ & 16.6 & $-25.8(-26.0)$ & 16.0 \\
& $1 \mathrm{p}$ & $-11.4(-12.1)$ & 16.7 & $-17.4(-17.8)$ & 16.0 & $-21.9(-22.2)$ & 15.5 \\
& $1 \mathrm{~d}$ & $-6.9(-8.1)$ & 15.7 & $-12.7(-13.4)$ & 15.2 & $-17.1(-17.6)$ & 14.8 \\
& $2 \mathrm{~s}$ & $-5.2(-6.6)$ & 15.1 & $-10.9(-11.7)$ & 14.8 & $-15.2(-15.8)$ & 14.5 \\
& $2 \mathrm{p}$ & $\mathrm{n}(-1.9)$ & $\mathrm{n}$ & $-4.8(-6.1)$ & 13.5 & $-8.9(-9.8)$ & 13.4 \\
& $2 \mathrm{~d}$ & $\mathrm{n}(\mathrm{n})$ & $\mathrm{n}$ & $\mathrm{n}(-0.7)$ & $\mathrm{n}$ & $-2.2(-3.7)$ & 11.9 \\
\hline \hline
\end{tabular}

Table 1: $\phi$-nucleus single-particle energies $E$ and half widths $\Gamma / 2$, for three values of $\Lambda_{K}$. When only the real part of the potential is included, the corresponding single-particle energy $E$ is given in parenthesis and $\Gamma=0$ for all nuclei. " $n$ " indicates that no bound state is found. All quantities are given in MeV.

\section{Summary and discussion}

We have calculated the $\phi$ meson mass and width in nuclear matter within an effective Lagrangian approach. The in-medium kaon masses are calculated in the QMC model, where the scalar and vector meson mean fields couple directly to the light $u$ and $d$ quarks (antiquarks) in the $K(\bar{K})$ mesons. At normal nuclear matter density we have found a downward shift of the $\phi$ mass of a few percent. On the other hand, the decay width has been broadened by an order of magnitude. We have also calculated the $\phi$-nucleus bound state energies and absorption widths for various nuclei by solving the Klein-Gordon equation with complex potentials. We expect that the $\phi$ should form bound states for all four nuclei selected, provided that the $\phi$ is produced in (nearly) recoilless kinematics. This feature is even more obvious in the case where the absorptive part of the potential is ignored. Given the similarity of the binding energies and widths, the signal for the formation of the $\phi$-nucleus bound states may be difficult to identify experimentally. Therefore, the feasibility of observation of the $\phi$-nucleus bound states needs further investigation, including explicit reaction cross section estimates.

This work was partially supported by Conselho Nacional de Desenvolvimento Científico e Tecnológico-CNPq, Grants No. 152348/2016-6 (J.J.C-M.), No. 400826/2014-3 and No. 308088/20158 (K.T.), No. 305894/2009-9 (G.K.), and No. 313800/2014-6 (A.W.T.), and Fundação de Amparo à Pesquisa do Estado de São Paulo-FAPESP, Grants No. 2015/17234-0 (K.T.) and No. 2013/01907-0 (G.K.). This research was also supported by the University of Adelaide and by the Australian Research Council through the ARC Centre of Excellence for Particle Physics at the Terascale (CE110001104), and through Grant No. DP151103101 (A.W.T.). 


\section{References}

[1] R. S. Hayano and T. Hatsuda, Rev. Mod. Phys. 82, 2949 (2010); S. Leupold, V. Metag and U. Mosel, Int. J. Mod. Phys. E 19, 147 (2010); A. Hosaka, T. Hyodo, K. Sudoh, Y. Yamaguchi and S. Yasui, Prog. Part. Nucl. Phys. 96, 88 (2017); G. Krein, A. W. Thomas and K. Tsushima, arXiv: 1706.02688 ; V. Metag, M. Nanova and E. Y. Paryev, Prog. Part. Nucl. Phys. 97, 199 (2017).

[2] T. Appelquist and W. Fischler, Phys. Lett. 77B, 405 (1978); S. J. Brodsky, I. A. Schmidt and G. F. de Teramond, Phys. Rev. Lett. 64, 1011 (1990); M. E. Luke, A. V. Manohar and M. J. Savage, Phys. Lett. B 288, 355 (1992); H. Gao, T. S. H. Lee and V. Marinov, Phys. Rev. C 63, 022201 (2001);

S. R. Beane, E. Chang, S. D. Cohen, W. Detmold, H.-W. Lin, K. Orginos, A. Parreo and M. J. Savage, Phys. Rev. D 91, no. 11, 114503 (2015); H. Gao, H. Huang, T. Liu, J. Ping, F. Wang and Z. Zhao, Phys. Rev. C 95, no. 5, 055202 (2017).

[3] A. I. Titov, Y. s. Oh and S. N. Yang, Phys. Rev. Lett. 79, 1634 (1997); A. I. Titov, Y. s. Oh, S. N. Yang and T. Morii, Phys. Rev. C 58, 2429 (1998); Y. s. Oh, A. I. Titov, S. N. Yang and T. Morii, Phys. Lett. B 462, 23 (1999); Y. s. Oh and H. C. Bhang, Phys. Rev. C 64, 055207 (2001); P. Gubler and K. Ohtani, Phys. Rev. D 90, no. 9, 094002 (2014).

[4] D. Kawama et al., PoS Hadron 2013, 178 (2013); D. Kawama et al., JPS Conf. Proc. 1, 013074 (2014); Y. Morino et al., JPS Conf. Proc. 8, 022009 (2015); T. Ishikawa et al., Phys. Lett. B 608, 215 (2005); R. Muto et al., Phys. Rev. Lett. 98, 042501 (2007); T. Mibe et al., Phys. Rev. C 76, 052202 (2007); X. Qian et al., Phys. Lett. B 680, 417 (2009); M. H. Wood et al., Phys. Rev. Lett. 105, 112301 (2010); A. Polyanskiy et al., Phys. Lett. B 695, 74 (2011).

[5] H. Ohnishi et al., Acta Phys. Polon. B 45, 819 (2014); P. Buhler et al. 2010 http: //j-parc.jp/researcher/Hadron/en/pac_0907/pdf/Ohnishi.pdf; P. Buhler et al. 2010 http: / / j-parc.jp/researcher/Hadron/en/pac_1007/pdf/ KEK_J-PARC-PAC2010-02.pdf

[6] M. Paolone et al. 2014 https://www.jlab.org/exp_prog/PACpage/PAC42/PAC42_FINAL_Report.pdf.

[7] C. M. Ko, P. Levai, X. J. Qiu and C. T. Li, Phys. Rev. C 45, 1400 (1992); T. Hatsuda and S. H. Lee, Phys. Rev. C 46, no. 1, R34 (1992); F. Klingl, T. Waas and W. Weise, Phys. Lett. B 431, 254 (1998); E. Oset and A. Ramos, Nucl. Phys. A 679 (2001) 616; D. Cabrera and M. J. Vicente Vacas, Phys. Rev. C 67, 045203 (2003); P. Gubler and W. Weise, Phys. Lett. B 751, 396 (2015); D. Cabrera, A. N. Hiller Blin and M. J. Vicente Vacas, Phys. Rev. C 95, no. 1, 015201 (2017).

[8] J. J. Cobos-Martínez, K. Tsushima, G. Krein and A. W. Thomas, Phys. Lett. B 771, 113 (2017).

[9] J. J. Cobos-Martínez, K. Tsushima, G. Krein and A. W. Thomas, Phys. Rev. C 96, no. 3, 035201 (2017).

[10] K. Tsushima, K. Saito, A. W. Thomas and S. V. Wright, Phys. Lett. B 429, 239 (1998) Erratum: Phys. Lett. B 436, 453 (1998).

[11] F. Klingl, N. Kaiser and W. Weise, Z. Phys. A 356, 193 (1996).

[12] K. Saito, K. Tsushima and A. W. Thomas, Prog. Part. Nucl. Phys. 58, 1 (2007).

[13] K. Saito, K. Tsushima and A. W. Thomas, Nucl. Phys. A 609, 339 (1996).

[14] K. Saito, K. Tsushima and A. W. Thomas, Phys. Rev. C 56, 566 (1997). 